\title{
Molecular characteristics of Brucella melitensis isolates from humans in Qinghai Province, China
}

\author{
Zhi-Jun Zhao ${ }^{1}$, Ji-Quan Li ${ }^{3}$, Li Ma ${ }^{1}$, Hong-Mei Xue ${ }^{1}$, Xu-Xin Yang ${ }^{1}$, Yuan-Bo Zhao ${ }^{1}$, Yu-Min Qin ${ }^{1}$, Xiao-Wen Yang ${ }^{2}$, \\ Dong-Ri Piao ${ }^{2}$, Hong-Yan Zhao ${ }^{2}$, Guo-Zhong Tian², Qiang Li ${ }^{1}$, Jian-Ling Wang ${ }^{1}$, Guang Tian ${ }^{1}$, Hai Jiang ${ }^{1,2^{*}}$ and \\ Li-Qing $X u^{1 *}$
}

\begin{abstract}
Background: The prevalence of human brucellosis in Qinghai Province of China has been increasing rapidly, with confirmed cases distributed across 31 counties. However, the epidemiology of brucellosis transmission has not been fully elucidated. To characterize the infecting strains isolated from humans, multiple-locus variable-number tandem repeats analysis (MLVA) and whole-genome single-nucleotide polymorphism (SNP)-based approaches were employed.

Methods: Strains were isolated from two males blood cultures that were confirmed Brucella melitensis positive following biotyping and MLVA. Genomic DNA was extracted from these two strains, and whole-genome sequencing was performed. Next, SNP-based phylogenetic analysis was performed to compare the two strains to 94 B. melitensis strains (complete genome and draft genome) retrieved from online databases.

Results: The two Brucella isolates were identified as B. melitensis biovar 3 (QH2019001 and QH2019005) following conventional biotyping and were found to have differences in their variable number tandem repeats (VNTRs) using MLVA-16. Phylogenetic examination assigned the 96 strains to five genotype groups, with QH2019001 and QH2019005 assigned to the same group, but different subgroups. Moreover, the QH2019005 strain was assigned to a new subgenotype, $I \mathrm{j}$, within genotype II. These findings were then combined to determine the geographic origin of the two Brucella strains.

Conclusions: Utilizing a whole-genome SNP-based approach enabled differences between the two B. melitensis strains to be more clearly resolved, and facilitated the elucidation of their different evolutionary histories. This approach also revealed that QH2019005 is a member of a new subgenotype (llj) with an ancient origin in the eastern Mediterranean Sea.
\end{abstract}

Keywords: Brucella melitensis, Multiple-locus variable-number tandem repeats analysis, Whole-genome sequencing, Single-nucleotide polymorphism

*Correspondence: jianghai@icdc.cn; qhxlq2006@163.com

${ }^{\dagger}$ Zhi-Jun Zhao and Ji-Quan Li contributed equally to this work ${ }^{1}$ Qinghai Institute for Endemic Disease Prevention and Control, The department of brucellosis prevention and control, Xining 810021, Qinghai, China

Full list of author information is available at the end of the article

\section{Background}

Brucellosis, which is caused by bacteria in the Brucella genus, is one of the most important zoonoses worldwide and is considered a "forgotten, neglected zoonosis" by the World Health Organization [1]. This disease is endemic in regions within Africa, Asia, Latin America and other original author(s) and the source, provide a link to the Creative Commons licence, and indicate if changes were made. The images or other third party material in this article are included in the article's Creative Commons licence, unless indicated otherwise in a credit line to the material. If material is not included in the article's Creative Commons licence and your intended use is not permitted by statutory regulation or exceeds the permitted use, you will need to obtain permission directly from the copyright holder. To view a copy of this licence, visit http://creativecommons.org/licenses/by/4.0/. The Creative Commons Public Domain Dedication waiver (http://creativeco mmons.org/publicdomain/zero/1.0/) applies to the data made available in this article, unless otherwise stated in a credit line to the data. 
countries along the Mediterranean Sea [2, 3]. Human infections can occur due to the consumption of contaminated non-pasteurized milk or cheese, or by occupational exposure to infected animals or their carcasses, uterine secretions, or aborted fetuses [4]. While the mortality rate of brucellosis is low, the morbidity rate is much higher. Worldwide, incidence of human brucellosis varies widely, from $<0.01$ to $>200$ per 100000 people in endemic disease areas [5]. In the mainland of China, the total incidence rate of human brucellosis increased from 0.92 per 100000 people in 2004 to 4.2 per 100000 people in 2014 [6]. Recently, in Qinghai Province, the incidence rate has increased from 0.04 per 100000 people in 2011 to 1.96 per 100000 people in 2018, with confirmed cases distributed across 31 counties within the entire province. Thus, brucellosis is becoming a major public health problem that impacts human physical and mental health.

Brucella is a gram-negative, facultative, intracellular pathogen that causes brucellosis in both humans and animals. Currently, the Brucella genus contains 12 accepted species, with four of those, namely, B. melitensis, B. abortus, B. suis and B. canis are considered zoonotic as they regularly cause humans infection [7]. While various species differ in host preference, virulence and/or zoonotic potential, most Brucella maintain a $97-99 \%$ genomic sequence identity $[8,9]$.

In humans, $B$. melitensis is the most virulent species and has been the predominant species associated with human brucellosis in China from 1953 to 2013 [10]. To ensure accurate epidemiological surveillance and to distinguish infected and vaccinated individuals, species identification and subtyping is essential [11]. Multiple loci variable-number tandem repeat analysis (MLVA) can provide the epidemiological relatedness among Brucella strains [12]. In a study examining Brucella in the Qinghai-Tibet Plateau region, none of the genotypes matched any of the sequences in the Brucella MLVA database (2012), possibly due to these strains having unique geographical characteristics or due to $B$. melitensis being the predominant species in that area [13]. Moreover, Brucella studies have also utilized whole-genome sequencing (WGS), which provides excellent genetic resolution, to resolve differences in closely related species [14]. In this study, we performed an MLVA typing of a collection of strains from the Qinghai province, 2018-2019. We selected two strains showing very distinct MLVA profiles for analysis using whole-genome single nucleotide polymorphism (SNP)-based approaches to elucidate the characteristics and homologies for epidemiological purposes. These findings will provide further insight into Brucella epidemiology and enable improved control and prevention of brucellosis in Qinghai Province.

\section{Methods}

\section{Bacterial strains}

Positive blood cultures were examined for brucellosis at the Qinghai Institute for Endemic Disease Prevention and Control in Qinghai Province, China, and deemed Brucella-positive between 2018 and 2019. The strains were identified as B. melitensis based on morphology and conventional identification methods according to standard biotyping procedures, including $\mathrm{CO}_{2}$ requirement, inhibition of growth by basic fuchsin and thionin, agglutination with monospecific antisera $(\mathrm{A}, \mathrm{M})$ and phage typing $\left(\mathrm{Bk}_{2}, \mathrm{~Tb}\right)$.

Total genomic DNA was extracted from the two $B$. melitensis strains, QH2019001 and QH2019005, using a Wizard Genomic DNA Purification Kit (Promega, city, USA) according to the manufacturer's instructions. The obtained DNA was then assessed via agarose gel electrophoresis and quantified using a Qubit 2.0 Fluorometer (Thermo Scientific, city, USA).

\section{Brucella MLVA-16 genotyping scheme}

MLVA-16 was performed as previously described [14]. All data were analyzed using BioNumerics (version 5.1; Applied Maths, Sint-Martens-Latem, Belgium). Clustering analysis was based on the categorical coefficient and the unweighted pair group method using the arithmetic averages (UPGMA) method [15].

\section{Whole-genome sequencing and single-nucleotide polymorphism analyses}

Sequencing libraries were generated using a NEBNext ${ }^{\circledR}$ Ultra $^{\mathrm{TM}}$ DNA Library Prep Kit for Illumina (NEB, city, USA) following the manufacturer's recommendations. WGS for the QH2019001 and QH2019005 strains was performed by Novogene Bioinformatics Technology Co., Ltd. (Beijing, China) using an Illumina NovaSeq 6000 (PE $150 \mathrm{bp}$ ). The raw data generated using the Illumina sequencing platform were assembled using SOAPdenovo (version 2.04). The obtained genomic sequences were available in GenBank under accession numbers of GCA 016411965.1 and GCA 016806105.1.

Genomic alignments between a sample genome and reference genome (or among more than two sample genomes) were performed by Novogene Bioinformatics Technology Co., Ltd. (Beijing, China) using the MUMmer [16] and LASTZ $[17,18]$ tools. Additionally, $94 B$. melitensis genomes (Additional file 1: Table S1) were retrieved from GenBank and used for comparison and preliminary phylogenetic analyses. A phylogenetic tree was constructed using TreeBeST and PHYML (maximum-likelihood based, with 1000 bootstrap replicates utilized) $[19,20]$. 


\section{Results}

Identification of Brucella strains

The Brucella strains QH2019001 and QH2019001 were identified as $B$. melitensis biovar 3 using a conventional biotyping method (Table 1).

\section{MLVA-16 genotyping results}

The MLVA-16 assay was used to determine the genotypes for 40 B. melitensis strains from human patients in Qinghai province, in 2018-2019. Two strains, QH2019001 (1-43-13-2-2-3-2-4-40-8-6-4-3-18-5) and QH2019005 (1-5-3-13-120 3-2-3-2-4-38-8-5-4-7-16-5) stand alone in the clustering analysis shown in Fig. 1, and define new MLVA-11 genotypes. For the two B. melitensis strains, variable number tandem repeat (VNTR) differences were present in bruce 08 and bruce 42 (panel 1) and bruce19, bruce 04 , bruce 09 and bruce 16 (panels $2 \mathrm{~A}$ and $2 \mathrm{~B}$ ). When combining these findings with epidemiological data, B. melitensis strain QH2019001 appears to have been imported from Anhui Province, while the QH2019005 strain appears to be native to Qinghai Province (Fig. 1).

\section{Genomic characteristic and phylogenetic analysis based on whole-genome SNPs}

The total contig sequence lengths were 3291786 bp for QH2019001 and 3289996 bp for QH2019005, with both having a GC content of $57.2 \%$. To gain insight into the geographical distributions of the 96 examined $B$. melitensis strains (including standard strains and $B$. melitensis strains around the world), a phylogenetic

Table 1 Biotyping identification results for the two Brucella melitensis strain

\begin{tabular}{|c|c|c|c|c|c|c|c|}
\hline \multirow[t]{2}{*}{ Strain IDs } & \multirow[t]{2}{*}{$\mathrm{CO}_{2}$ Requirement } & \multicolumn{2}{|l|}{ Sensitivity to dyes } & \multicolumn{2}{|c|}{ Serum agglutination } & \multicolumn{2}{|l|}{ RTD } \\
\hline & & Fuchin 1:25000 & Thionin 1:50 000 & A & M & BK2 & $\mathrm{Tb}$ \\
\hline QH2019001 & - & + & + & + & + & + & + \\
\hline QH2019005 & - & + & + & + & + & + & + \\
\hline
\end{tabular}

RTD Routine test dilution, BK2 Berkeley, Tb Tbilisi

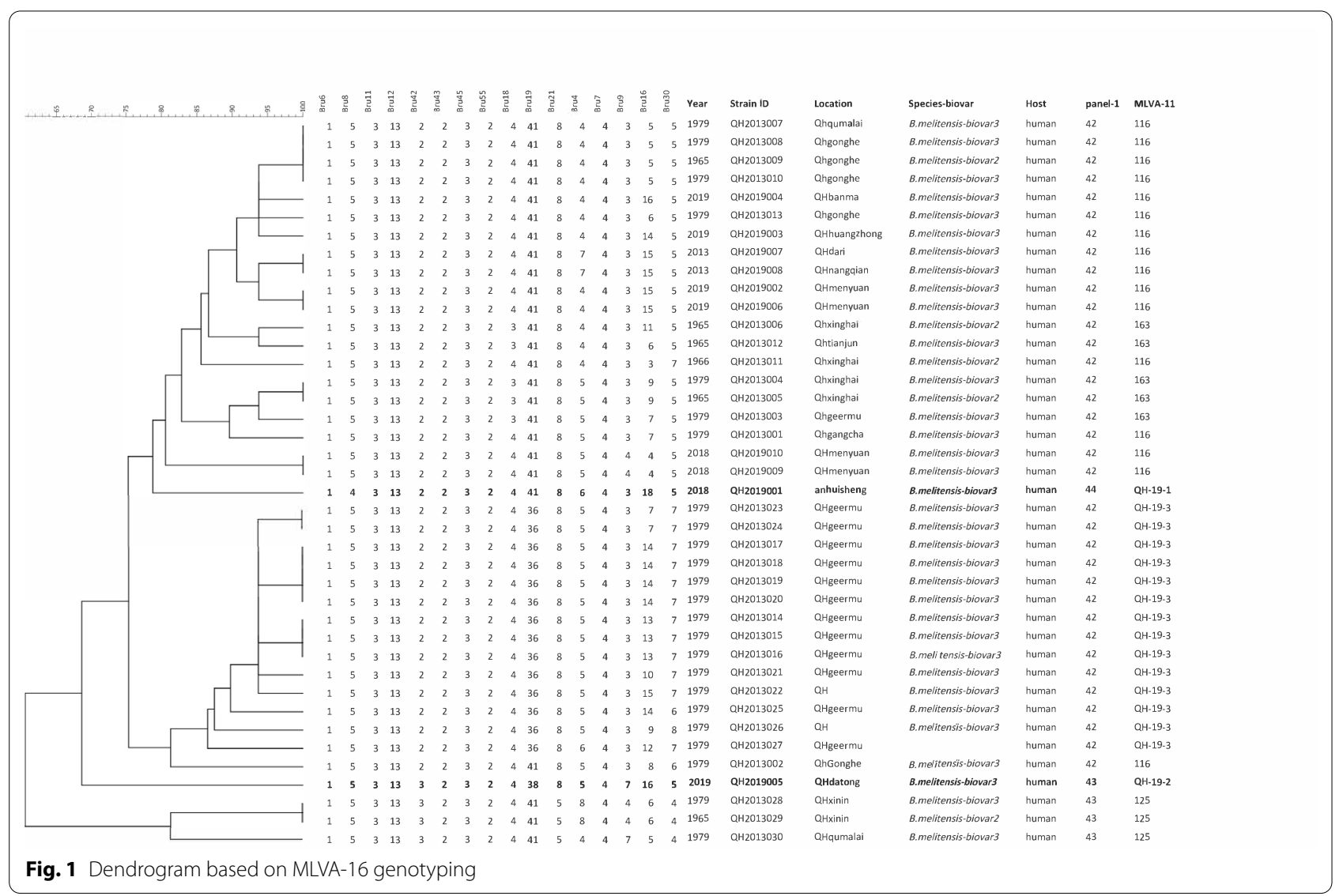


analysis was performed using the whole-genome SNPs. The strains were divided into five major genotypes as follows: Genotype I corresponds to the West Mediterranean clade, Genotype II to the East Mediterranean clade, and genotypes III to V belong to the Americas group. Genotype III was recently designated as the African clade (strain 65/112) by Foster et al. [21] (Fig. 2). The $16 \mathrm{M} 22$ strain was isolated from an infected mouse that

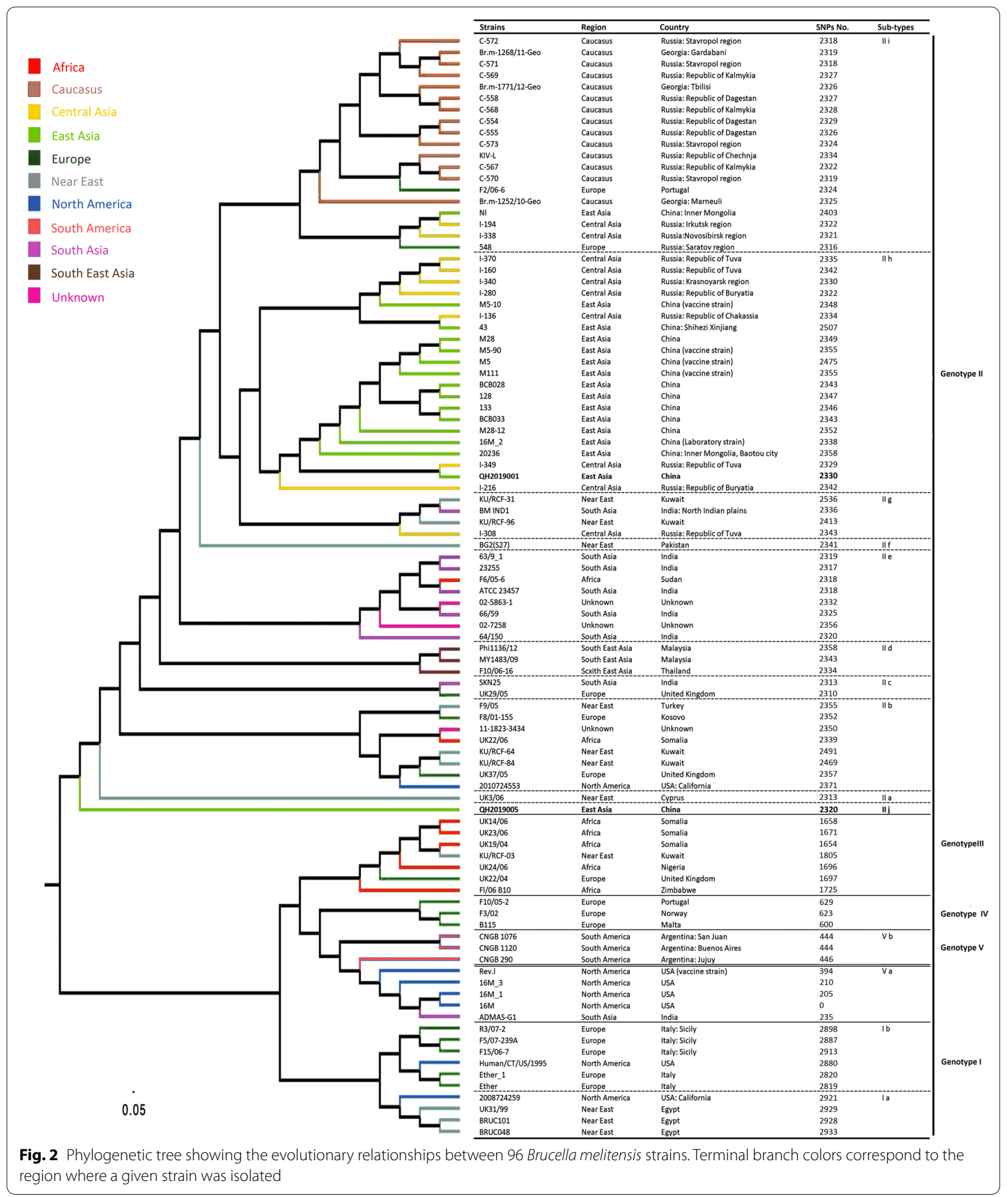


was reported to have accumulated mutations during the infection. The position in Fig. 2 shows clearly that it is not derived from $16 \mathrm{M}$. The QH2019001 and QH2019005 strains were both associated with the genotype II group. Furthermore, several clades and subclades were isolated within the lineages and were associated with geographic attributes. The QH2019001 strain was assigned to subgenotype IIh, while the QH2019005 strain was assigned to subgenotype IIj (a new subgroup).

\section{Discussion}

MLVA genotyping provides valuable insight when examining an epidemiological linkage or trace-back investigation during a brucellosis outbreak [22-24]. Based on the MLVA-11, the QH2019001 and QH2019005 strains formed unique genotypes and represented as single independent strain, which suggests that these two strains were not related epidemiologically. Thus the exact origins of the two strains could not be determined by MLVA. However, WGS-based analysis has been shown to distinguish very closely related $B$. melitensis strains and can discriminate intraspecies relationships [25, 26]. In this study, whole-genome SNP analysis showed that the QH2019001 and QH2019005 strains were both assigned to genotype II, but to different subgroups. Furthermore, B. melitensis strain QH2019005 was assigned to genotype IIj (a new subtype), which is closely associated with subgenotype IIa that contained only a single strain, UK3/06. The QH2019001 strain was assigned to subgenotype IIh. The differences between the QH2019001 and QH2019005 strains suggest that the nucleotide variation may be attributed to changes in geographic distribution. It may have been driven by the relatively isolated and more unique environment of the Qinghai-Tibet Plateau, with its extremely high altitudes. Due to the environment, it is difficult for lowland livestock breeds or wild animals to survive; hence, livestock exchange between Qinghai Province and other regions would be limited [13]. Further investigation of the movement of sheep should be performed to identify the origins of these strains.

The QH2019005 strain was the most similar to the UK3/06 strain that was isolated from the Near East, thus suggesting that this strain and its new subgenotype have ancient origins. The QH2019001 strain was the most similar to the I-349 strain that was isolated from Central Asia (Russia: Republic of Tuva) [27], thus suggesting that this strain shares a common origin. Furthermore, active trade between Russia and China could have promoted the transmission of B. melitensis strain QH2019001 to other regions (Anhui Province) in China.
In summary, we observed that strains in this region exhibited unique characteristics of origin and evolution. Further efforts, examination of more strains, WGS, and collection of epidemiological data from the neighboring provinces are needed to accurately outline the pattern of transmission of brucellosis in Qinghai, China.

\section{Conclusion}

In the present study, the molecular characteristics of two human Brucella strains isolated from Qinghai Province were examined to gain a further understanding of the epidemiology of brucellosis. The two strains were found to have different origins and evolutionary histories, with the native strain, QH2019005, assigned to a new subgenotype with an ancient origin in the eastern Mediterranean region. Additionally, this study further highlighted that utilizing a whole-genome SNP-based approach can enable intraspecies relationships between $B$. melitensis to be more fully examined.

\section{Abbreviations}

WGS: Whole-genome sequencing; MLVA: Multiple loci variable-number tandem repeat analysis; SNP: Single nucleotide polymorphism; SAT: Serum agglutination tests; VNTR: Variable number tandem repeat.

\section{Supplementary Information}

The online version contains supplementary material available at https://doi. org/10.1186/s40249-021-00829-0.

Additional file 1: Table S1. B. melitensis genome information.

Acknowledgements

Not applicable.

Authors' contributions

Conceptualization: $\mathrm{HJ}$ and LX. Formal analysis: ZZ and JL. Investigation: $Z Z$, JL, LM, HX, XY, YZ, YQ, GT, JW, and QL. Methodology: DP, HZ, GT, and XY. Project administration: $H J$ and LX. Supervision: LX. Writing-original draft: ZZ and JL. Writing-review \& editing: HJ. All authors read and approved the final manuscript.

\section{Funding}

This study was supported by National Key R\&D Program of China (Grant No. 2020YFA0907101), Major Infectious Diseases such as AIDS and Viral Hepatitis Prevention and Control Technology Major Projects (2018ZX10201002) and the National Natural Scientific Foundation of China (81860588).

Availability of data and materials

All the relevant data have been provided within the manuscript or as a supporting file.

\section{Declarations}

Ethics approval and consent to participate

This research was conducted according to the principles of the Declaration of Helsinki, and the study protocol was approved by the Ethics Committees of the National Institute for Communicable Disease Control and Prevention (Beijing, China). Informed consent was obtained from the two patients who 
received a brucellosis diagnosis at the Qinghai Institute for Endemic Disease Prevention and Control based on the Diagnosis for Brucellosis Standards (WS 269-2019) used in China. Obtained patient history and data were anonymized for the purpose of this study. Blood samples were obtained and Brucella strains were isolated.

\section{Consent for publication}

Not applicable.

\section{Competing interests}

The authors have no competing interests to declare.

\section{Author details}

' Qinghai Institute for Endemic Disease Prevention and Control, The department of brucellosis prevention and control, Xining 810021, Qinghai, China. ${ }^{2}$ State Key Laboratory for Infectious Disease Prevention and Control, Collaborative Innovation Center for Diagnosis and Treatment of Infectious Diseases, National Institute for Communicable Disease Control and Prevention, Chinese Center for Disease Control and Prevention, Beijing, China. ${ }^{3}$ Key Laboratory of Plaque Prevention and Research, Qinghai Institute for Endemic Disease Prevention and Control, National Health Commission (2019PT310004) and Key Laboratory for Plague Prevention and Control of Qinghai Province, Xining 810021, Qinghai, China.

\section{Received: 22 September 2020 Accepted: 19 March 2021}

Published online: 26 March 2021

\section{References}

1. Zhou K, Wu BB, Pan H, Paudyal N, Jiang J, et al. One health approach to address zoonotic BRUCELLOSis: a spatiotemporal associations study between animals and humans. Front Vet Sci. 2020;7:521. https://doi.org/ 10.3389/fvets.2020.00521.

2. Tuon FF, Gondolfo RB, Cerchiari NC. Human-to-human transmission of Brucella - a systematic review. Trop Med Int Health. 2017;22:539-46. https://doi.org/10.1111/tmi.12856.

3. Rubin B, Band JD, Wong P, Colville J. Person-to-person transmission of Brucella melitensis. Lancet. 1991;337(8732):14-5. https://doi.org/10.1016/ 0140-6736(91)93332-4

4. Pappas G, Papadimitriou P, Akritidis N, Christou L, Tsianos EV. The new global map of human brucellosis. Lancet Infect Dis. 2006;6:91-9. https:// doi.org/10.1016/S1473-3099(06)70382-6

5. Mambres DH, Boarbi S, Michel P, Bouker N, Escobar-Calle L, Desqueper D, et al. Imported human brucellosis in Belgium: bio and molecular typing of bacterial isolates, 1996-2015. PLoS ONE. 2017;12:e0174756. https://doi. org/10.1371/journal.pone.0174756.

6. Lai S, Zhou H, Xiong WY, Gilbert M, Yu HJ, Huang ZJ, et al. Changing epidemiology of human brucellosis, China, 1955-2014. Emerg Infect Dis. 2017:23:184-94. https://doi.org/10.3201/eid2302.151710.

7. Plumb GE, Olsen SC, Buttke D. Brucellosis: 'one health' challenges and opportunities. Rev Sci Tech. 2013;32:271-8. https://doi.org/10.20506/rst. 32.1.2195.

8. Delvecchio VG, Kapatral V, Redkar RJ, Patra G, Mujer C, Los T, et al. The genome sequence of the facultative intracellular pathogen Brucella melitensis. Proc Natl Acad Sci U S A. 2002;99:443-8. https://doi.org/10. 1073/pnas.221575398.

9. Zaki NA, Salloum T, Osman M, Rafei R, Hamze M, Tokajian S. Typing and comparative genome analysis of Brucella melitensis isolated from Lebanon. FEMS Microbiol Lett. 2017;364:fnx199. https://doi.org/10.1093/ femsle/fnx199.

10. Tian GZ, Cui BY, Piao DR, Zhao HY, Li LY, Liu X, et al. Multi-locus variablenumber tandem repeat analysis of Chinese Brucella strains isolated from
1953 to 2013. Infect Dis Poverty. 2017;6:89. https://doi.org/10.1186/ s40249-017-0296-0.

11. Jiang $H$, Wang $H, X u L Q, H u G Y, M a J Y, X i a o ~ P$, et al. MLVA genotyping of Brucella melitensis and Brucella abortus isolates from different animal species and humans and identification of Brucella suis vaccine strain S2 from cattle in China. PLoS ONE. 2013;8:e76332. https://doi.org/10.1371/journal. pone.0076332.

12. Flèche $P L$, Jacques I, Grayon $M$, Dahouk $S A$, Bouchon $P$, Denoeud F, et al. Evaluation and selection of tandem repeat loci for a Brucella MLVA typing assay. BMC Microbiol. 2006;6:9. https://doi.org/10.1186/1471-2180-6-9.

13. Ma JY, Wang $H$, Zhang XF, Xu LQ, Hu GY, Jiang $H$, et al. MLVA and MLST typing of Brucella from Qinghai China. Infect Dis Poverty. 2016;4:1-8. https://doi.org/10.1186/s40249-016-0123-z.

14. Sankarasubramanian J, Vishnu US, Gunasekaran P, Rajendhran J. A genome-wide SNP based phylogenetic analysis distinguishes different biovars of Brucella suis. Infect Genet Evol. 2016;41:213-7. https://doi.org/ 10.1016/j.meegid.2016.04.012.

15. Hua GJ, Hung CL, Lin CY, Wu FC, Chen YW, et al. MGUPGMA: a fast UPGMA algorithm with multiple graphics processing units using NCCL. Evol Bioinform. 2017:3:1176934317734220. https://doi.org/10.1177/1176934317 734220.

16. Marçais G, Delcher AL, Phillippy AM, Coston R, Salzberg SL, Zimin A. MUMmer4: a fast and versatile genome alignment system. PLoS Comput Biol. 2018;14:e1005944. https://doi.org/10.1371/journal.pcbi.1005944.

17. Li H, Handsaker B, Wysoker A, Fennell T, Ruan J, Homer N, et al. The sequence alignment/map format and SAMtools. Bioinformatics. 2009;25(16):2078-9. https://doi.org/10.1093/bioinformatics/btp352.

18. Altschul SF, Gish W, Miller W, Myers EW, Lipman DJ. Basic local alignment search tool. J Mol Biol. 1990;215(3):403-10. https://doi.org/10.1016/ S0022-2836(05)80360-2.

19. Guindon S, Dufayard JF, Lefort V, Anisimova M, Hordijk W, Gascuel O. New algorithms and methods to estimate maximum-likelihood phylogenies: Assessing the performance of PhyML 3.0. Syst Biol. 2010;59:307-21.

20. Vilella AJ, Severin J, Ureta-Vidal A, Heng L, Durbin R, Birney E. EnsemblCompara GeneTrees: Complete, duplication-aware phylogenetic trees in vertebrates. Genome Res. 2008:19:327-35.

21. Foster JT, Walker FM, Rannals BD, Hussain MH, Drees KP, Tiller RV, et al. African lineage Brucella melitensis isolates from Omani livestock. Front Microbiol. 2018;8:2702. https://doi.org/10.3389/fmicb.2017.02702.

22. Liu ZH, Wang LJ, Piao DR, Wang M, Liu RH, Zhao HY, et al. Molecular investigation of the transmission pattern of Brucella suis 3 from Inner Mongolia China. Front Vet Sci. 2018:5:271. https://doi.org/10.3389/fvets.2018.00271.

23. Zhang FB, Li ZW, La XL, Ma XM, Zhang YX, Ji P, et al. Multiple-locus variable-number tandem-repeat analysis of Brucella isolates from patients in Xinjiang China. Int J Clin Exp Med. 2015;8(9):15716-23.

24. Kattar MM, Jaafar RF, Araj GF, Fleche FL, Matar GM, et al. Evaluation of a multilocus variable-number tandem-repeat analysis scheme for typing human Brucella isolates in a region of brucellosis endemicity. Clin Microbiol. 2008:46(12):3935-40. https://doi.org/10.1128/JCM.00464-08.

25. Georgi E, Walter MC, Pfalzgraf MT, Northoff BH, Holdt LM, et al. Whole genome sequencing of Brucella melitensis isolated from 57 patients in Germany reveals high diversity in strains from Middle East. PLoS ONE. 2017:12(4):e0175425. https://doi.org/10.1371/journal.pone.0175425.

26. Janowicz A, Massis FD, Ancora M, Cammà C, Patavino C, et al. Core genome multilocus sequence typing and single nucleotide polymorphism analysis in the epidemiology of Brucella melitensis infections. J Clin Microbiol. 2018;56(9):e00517-e518. https://doi.org/10.1128/JCM. 00517-18.

27. Deniskova TE, Dotsev AV, Selionova MI, Kunz E, Medugorac L, et al. Population structure and genetic diversity of 25 Russian sheep breeds based on whole-genome genotyping. Genet Sel Evol. 2018;50:29. https://doi. org/10.1186/s12711-018-0399-5. 